\title{
A new blood test uses DNA methylation to detect and predict the spread of breast cancer
}

\author{
Martin Widschwendter
}

lona Evans

Allison Jones

Shohreh Ghazali

Daniel Reisel

Andy Ryan

Aleksandra Gentry-Maharaj

Michal Zikan

David Cibula

Johannes Eichner

Marianna Alunni-Fabbroni

Julian Koch

Wolfgang J. Janni

Tobias Paprotka

Timo Wittenberger

Usha Menon

Benjamin Wahl

Brigitte Rack

Harri Lempiäinen

\section{Video Abstract}

Keywords: Cell-free DNA, DNA methylation, Serum DNA, Breast cancer, Early diagnosis, Personalized treatment

Posted Date: November 21st, 2020

DOI: https://doi.org/10.21203/rs.3.rs-113115/v1

License: (9) This work is licensed under a Creative Commons Attribution 4.0 International License. Read Full License 


\section{Abstract}

Early detection of breast cancer can be the deciding factor between successful treatment and death. But mammography - the most common detection method - doesn't always find tumors before cancer has spread. Now, using only a sample of blood, an international research team has found a way to identify fatal breast cancers up to 12 months before they're usually diagnosed. The blood test can even find cancer before tumors become visible, creating new possibilities for early treatment. To develop the test, the researchers first identified DNA signatures specific to breast cancer. Cancer cells shed small pieces of DNA into the blood. This genetic material carries unique DNA methylation patterns. DNA methylation is an epigenetic modification, meaning it can alter gene function even when the gene's sequence stays the same. Because methylation patterns change very early in breast cancer development, they offer a promising detection tool. The team used computer software along with a special type of DNA sequencing to discover cancer-specific methylation patterns in 31 tissue samples. These patterns were then tested in blood samples from women with primary, metastatic, or no breast cancer. This narrowed down a methylation signature in a single region - deemed EFC\#93 - that acted as a marker for metastatic cancer. The researchers then looked for EFC\#93 methylation in blood samples from 925 healthy women who developed either fatal or non-fatal breast cancer within 3 years. The marker was found in $43 \%$ of the women with fatal breast cancer 3 to 6 months before they were diagnosed. In $25 \%$ of this group, it was found 6 to 12 months before diagnosis. These results suggest that even before a tumor is found, if EFC\#93 methylation is present in blood samples, breast cancer has likely already developed and started to spread. The team also studied blood samples from women with breast cancer before and after chemotherapy. The results showed that the treatment was far less effective for the women with EFC\#93 methylation, who were more likely to experience relapse and death than those without the marker. Although it's not yet clear whether looking at DNA methylation will lead to life-saving treatments, these findings show that earlier detection of aggressive cancers is possible. 